\title{
PROTEÍNAS TOTAIS DO LCR NO PROGNÓSTICO DO PACIENTE COM ACIDENTE VASCULAR ENCEFÁLICO HEMORRÁGICO
}

\author{
JOÃO BAPTISTA DOS REIS-FILHO*, SONIA BEATRIZ FELIX RIBEIRO**, YARA JULIANO ***
}

\begin{abstract}
RESUMO - O prognóstico dos pacientes com acidente vascular encefálico hemorrágico (AVEH) 6 reservado, apresentando altas taxas de morbidade e mortalidade. Estudos experimentais sugerem que existe alteraça da barreira hematoencefálica nos AVEH experimentais e que estas alteraçðes estâo relacionadas ao desenvolvimento do vascespasmo e pior prognóstico. Distúrbios da barreira hematoencefálica podem ser avaliados pelo estudo da taxa das proteínas totais do LCR. O propósito deste estudo é avaliar o valor da taxa de protefnas totais no prognóstico, em relaçăo à sobrevida, de pacientes com derrame de sangue no espaço subaracnóideo. Para tanto foram estudadas 254 fichas de pacientes do Departamento de Neurologia e Neurocirurgia da Escola Paulista de Medicina, com diagnóstico de AVEH, 144 do sexo masculino e 110 do sexo feminino, com idade variando de 8 a 84 anos. Foi verificado que a taxa de proteínas totais está diretamente correlacionada ao prognóstico, $\mathrm{em}$ relação à sobrevida, nos pacientes com AVEH. Quanto mais elevada a taxa de proteínas, pior o progn6stico; no material deste estudo todos os pacientes, em número de 15, que tiveram taxa de protefnas totais no LCR acima de $3000 \mathrm{mg} / 100 \mathrm{ml}$ faleceram. Este achado indica que a taxa de proteinas totais no LCR de paciente com AVEH é um preditor de óbito. A análise comparativa entre os sexos năo mostrou diferença estatística. Porém quando foram correlacionados o sexo, a idade e taxa de proteínas, verificou-se que homens acima de 50 anos tiveram taxa de proteínas mais elevadas que mulheres na mesma faixa etária, demonstrando que homens idosos têm pior prognóstico que as mulheres nessa mesma faixa etária.
\end{abstract}

PALAVRAS-CHAVE: acidente vascular encefálico hemorrágico, hemorragia subaracnóidea, liquido cefalorraqueano, prognóstico.

Total proteins level on CSF and their prognostic significance in subarachnoid hemorrhage

SUMMARY - The main purpose of this study was to verify the value of CSF total proteins level on the prognosis of subarachnoid hemorrhage. In order of this, samples of 254 patients with diagnosis of intracranial bleeding were analyzed, with special attention to the rate of CSF total proteins. Statistical tests for evaluation of the results have been accomplished, revealing a close relationship between the total proteins rates increase and death in patients with subarachnoid hemorrhage, independent of sex and age. The limit score of total proteins level for survive was $3000 \mathrm{mg} / 100 \mathrm{ml}$ (nephelometric method).

KEY WORDS: stroke, subarachnoid hemorhage, cerebrospinal fluid, prognosis.

As doenças cerebrovasculares representam importante causa de incapacidade física e 6 a $3^{2}$ causa de morte nos Estados Unidos da América',13. Na literatura atual existe grande controvérsia quanto à indicação da punção para coleta do líquido cefalomaquiano (LCR), no diagnóstico do

Resumo de Tese realizada no Setor de Líquido Cefalorraquiano, para obtençăo do Título de Mestre pelo Curso de Pós-graduação (PG) em Clínica Neurológica da Escola raulista de Medicina. (EPM): esta pesquisa foi parcialmente financiada pelo CNPq: *Professor Adjunto do Departamento de Neurologia e Neurocirurgia da EPM, Pesquisador do CNPq e Chefe do Setor de LCR; **Mestre pelo Curso de PG em Clínica Neurológica da EPM. ***Professora Assistente da Disciplina de Bioestatística da EPM. Aceite: 22-julho-1994.

Dr. Joāo Baptista dos Reis Filho. Setor de Líquido Cefalorraquiano, Disciplina de Neurologia, Escola Paulista de Medicina - Rua Botucatu 740 - 04023-900 São Paulo SP - Brasil 
acidente vascular encefálico ${ }^{15-17,20,33.35}$. Quando o exame de tomografia computadorizada craniana (TC) mostra-se normal, o exame do LCR pode ser de grande utilidade no diagnóstico da hemorragia intracraniana ${ }^{4.14 .16,19.22,33}$. Por outro lado, havendo necessidade de se ter um diagnóstico imediato e não havendo possibilidade da realização da TC, o exame do LCR pode ser realizado ${ }^{14,17,33,34}$. Diversos estudos tentam correlacionar as alteraçōes do LCR com o prognóstico $\mathrm{em}$ pacientes com acidente vascular encefálico hemorrágico, sem sucesso ${ }^{3,11,12}$. Chehrazi e Giri6 notaram que sangramento com mais de 400.000 hemácias/ $\mathrm{mm}^{3}$ no LCR relacionava-se com o desenvolvimento de vasoespasmo e com pior prognóstico do paciente com hemorragia subaracnóidea. Alguns estudos experimentais ${ }^{9,10,28.29}$ sugerem que existe alteração da barreira hematoencefálica nos acidentes vasculares encefálicos hemorrágicos experimentais e que estas alterações estão relacionadas ao desenvolvimento do vasoespasmo e pior prognóstico. Sugerem que essas mesmas alterações poderiam ocorrer em pacientes com hemorragia subaracnóidea espontânea. A taxa de proteínas totais no LCR é um meio de se avaliar o estado da barreira hematoencefálica ${ }^{26,38}$. O propósito deste estudo é avaliar o valor da taxa de proteínas totais no prognóstico, em relação à sobrevida, de pacientes com derrame de sangue no espaço subaracnóideo.

\section{MATERIAL E MÉTODO}

O material deste estudo é composto por 254 fichas de pacientes do Departamento de Neurologia e Neurocirurgia da Escola Paulista de Medicina, com diagnóstico de acidente encefálico vascular hemorrágico, nos quais foi feita coleta e exame do LCR. Destas fichas, 144 eram pacientes do sexo masculino e 110 do sexo feminino, com idade variando de 8 a 84 anos. Foram excluídos os casos de hemorragia de origem traumática, sendo consideradas apenas as hemorragias espontâneas, de etiologia vascular. Este material foi selecionado de um grupo de 363 pacientes, atendidos no período de janeiro- 1985 a julho-1990 com LCR hemorrágico. Foram excluídos deste estudo 109 pacientes por estar o prontuário incompleto ou extraviado.

As amostras de LCR foram obtidas através de punçăo lombar ou cisternal, sempre nas primeiras 48 horas, desde o início dos sintomas neurovasculares. O tempo fixado para observação da evolução dos pacientes em relação à sobrevida foi de 7 dias.

Em todas as amostras foi feito o exame de rotina no LCR, que consta de medida das pressões inicial e final, observação do aspecto e cor, antes e após centrifugação, contagem de células e de hemácias, dosagem das taxas de proteínas, cloretos e glicose, reaçð̋es imunobiológicas para diagnóstico da sífilis e da cisticercose. A taxa de proteínas foi determinada pela técnica nefelométrica de Denis \& Ayer ${ }^{8}$.

Os pacientes foram divididos em 5 grupos de acordo com a taxa de proteínas: Grupo A, 10 a $100 \mathrm{mg} / 100$ $\mathrm{ml}$; Grupo B, 101 a $500 \mathrm{mg} / 100 \mathrm{ml}$; Grupo C, 501 a $1.000 \mathrm{mg} / 100 \mathrm{ml}$; Grupo D, 1.001 a $3.000 \mathrm{mg} / 100 \mathrm{ml} \mathrm{e}$ Grupo E, acima de $3.000 \mathrm{mg} / 100 \mathrm{ml}$. Inicialmente foram comparados os números de óbitos presentes em cada grupo. Com a finalidade de avaliar a influência de outros fatores, foram feitas comparaçōes levando-se em consideraçāo o sexo e a faixa etária. Os pacientes foram subdivididos arbitrariamente: até 20 anos, de 21 a 50 anos e acima de 50 anos.

Para a análise dos dados estatísticos foram utilizados o teste da partição do quiquadrado ${ }^{7}$ para tabelas $2 \mathrm{XN}$, com o objetivo de comparar as percentagens de sobrevida nos diferentes grupos de taxa de proteínas; 0 teste de Mann-Whitney para duas amostras independente ${ }^{31}$ com o objetivo de comparar horiens e mulheres, tanto no grupo dos sobreviventes quanto no grupo dos que faleceram, e para comparar pacientes que morreram ou sobreviveram, em cada sexo, para cada grupo etário, em relação às taxas de proteínas totais no LCR; em alguns casos, levando-se em consideração o tamanho da amostra, este teste foi aplicado com aproximação à curva normal (estatística $z$ ); foi também utilizada a análise de variância por postos de Kruskal-Wallis ${ }^{31}$ para comparar, separadamente para homens e para mulheres, os diferentes grupos etários, em relaçăo às taxas de proteínas totais; quando mostrou diferença significante, esta análise foi complementada pelo teste de comparações múltiplas". Em todos os testes fixou-se em 0,05 ou $5 \%(<0,05)$ o nível para rejeição da hipótese de nulidade, assinalando-se com asterisco os valores significantes. 


\section{RESULTADOS}

Houve correlação estatística significante entre a taxa de proteínas totais do LCR e o número de pacientes que faleceram ou que sobreviveram. Assim, quanto maior a taxa de proteínas totais maior o número de óbitos. No material deste estudo, os pacientes com taxas de proteínas totais no LCR superiores a $3.000 \mathrm{mg} / 100 \mathrm{ml}$ não sobreviveram (Tabela 1).

Foram agrupados pacientes do sexo feminino e do masculino segundo a evolução e a taxa de proteinas em mg/100 ml. O teste não mostrou correlação entre sexo, prognóstico e taxa de proteínas (Tabela 2).

Quando foram comparados pacientes do sexo feminino e do masculino, segundo o grupo etário e taxa de proteínas, observaram-se valores significativamente maiores da taxa de proteínas para homens acima de $\mathbf{5 0}$ anos (Tabela 3).

Quando agrupados segundo a faixa etária, sexo, prognóstico e taxa de proteínas totais, houve significância estatística em relaçāo ao óbito e taxa de proteínas elevadas, em todos os grupos etários, tanto do sexo feminino quanto no masculino (Tabela 4).

Tabela 1. Correlação entre sobrevida e taxa de proteínas.

\begin{tabular}{ccccc}
\hline & \multicolumn{3}{c}{ Número de pacientes } & \\
\cline { 2 - 4 } $\begin{array}{c}\text { Taxa de proteŕnas } \\
\mathrm{mg} / 100 \mathrm{ml}\end{array}$ & sobrevida & 6bito & total & $\begin{array}{c}\text { Proporçăo } \\
\text { sobrevida }\end{array}$ \\
\hline \hline$<100$ & 72 & 3 & 75 & $(96 \%)$ \\
$100-500$ & 50 & 14 & 64 & $(78,1 \%)$ \\
$501-1000$ & 28 & 18 & 46 & $(60,9 \%)$ \\
$1001-3000$ & 14 & 40 & 54 & $(25,9 \%)$ \\
$>3000$ & 0 & 15 & 15 & $(0,0 \%)$ \\
\hline
\end{tabular}

Teste do Quiquadrado

$\mathrm{X}^{2}$ calculado $=100,4^{*}$

$X^{2}$ crítico $=9,5$

Participação do Quiquadrado

$\mathrm{X}^{2}$ crítico $=3,8$

1'. $[(1000-3000)+>3000] \times[<100+(100-500)+(500-1000)]$

$2^{2}$. ( $\left.>3000\right) \times(1000-3000)$

$\mathrm{X}^{2}=81,2^{*}$

$\mathrm{X}^{2}=3,4$

$3^{4} \cdot[(100-500)+(500-1000)] \times(<100)$

$X^{2}=12,3^{*}$

$4^{2} .(500-1000) \times(100-500)$

$\mathrm{X}^{2}=3,5$

Tabela 2. Correlação da taxa de proteínas, segundo o sexo e prognóstico.

\begin{tabular}{lcc}
\hline & \multicolumn{2}{c}{ Taxa de proténas (valor médio) $\mathrm{mg} / 100 \mathrm{ml}$} \\
\cline { 2 - 3 } & sobrevida & obito \\
\hline \hline Sexo masculino & 318 & 1675 \\
Sexo feminino & 402 & 1768 \\
\hline
\end{tabular}

Teste de Mann Whitney com aproximação à curva normal

(masculino $\mathrm{x}$ feminino)

$z$ crítico $=1,96$

sobreviventes

óbito 
Tabela 3. Correlação da taxa de proteinas segundo sexo e faixa etária.

\begin{tabular}{llccccccc}
\hline & \multicolumn{3}{c}{ Sexo masculino } & & \multicolumn{3}{c}{ Sexo feminino } \\
Faixa etária (anos) & até 20 & $21-50$ & $51 \mathrm{e}+$ & & até 20 & $21-50$ & $51 \mathrm{e}+$ \\
\hline \hline Taxa de proténas mg/100 ml (valor médio) & 563 & 675 & 1021 & & 1054 & 774 & 842 \\
Média da soma de postos & 58,5 & 66,5 & 82,8 & & 58,7 & 56,6 & 54,1 \\
\hline
\end{tabular}

Análise de variância por postos de Kruskall-Wallis

(Ate 20 anos $\times 21-50 \times 50$ anos e +)

H critico $=6,0$

Sexo masculino

H calculado $=6,5^{*}$
Sexo feminino

$\mathrm{H}$ calculado $=0,2$

Teste de comparaçōes múltiplas

20 - 50 anos $<50$ anos e +

Tabela 4. Correlafão entre sobrevida, grupo etário, sexo e taxa de proteinas.

Idade (anos)

Taxa de proteínas em mg/100 $\mathrm{ml}$ (Valor médio)

Sexo masculino

6bito sobrevida

$20-50$

1608

330

1732

328

$51-84$

Sexo feminino

1732

Teste de Mann Whitney

(6bito $x$ sobrevida)

Ate 20 anos

20 a 50 anos

51 a 84 anos

Número de casos insuficientes para Masculino: Feminino: análise estatística

1521

\begin{tabular}{cc}
\hline 6bito & sobrevida \\
1521 & 428 \\
1692 & 322 \\
\hline
\end{tabular}


sexo, idade e taxa de proteínas, verificou-se que homens acima de 50 anos tiveram taxa de proteinas mais elevadas que mulheres na mesma faixa etária, demonstrando que homens idosos têm pior prognóstico do que as mulheres nessa mesma faixa etária (Tabela 4). Esta associação já foi observada por alguns autores ${ }^{5.30}$, porém muitos acreditam que a idade não é fator de influência no prognóstico dos acidentes vasculares encefálicos hemorrágicos $3,21,25$.

Estudos experimentais ${ }^{9,10,28,29}$ demonstram que há correlação entre o aumento da barreira hematencefálica nos acidentes vasculares encefálicos e pior prognóstico. Este estudo confirma os achados experimentais visto que a taxa de proteínas totais é um dos indicadores da lesæa da barreira hematencefálica.

Da mesma forma como evidenciado por este estudo, taxas de proteínas elevadas têm sido correlacionadas com pior prognóstico em pacientes com traumatismo crânio-encefálico ${ }^{27}$, meningite bacteriana aguda ${ }^{37}$ e meningencefalite tuberculosa ${ }^{36}$.

Sendo a taxa de proteínas totais do LCR um parâmetro de avaliaçæo da barreira hematencefálica, quanto maior a taxa de proteínas, maior será o dano encefálico. Esta associação lógica explica porque taxas de proteínas elevadas no LCR estão associadas a piores prognósticos, nåo só para acidentes vasculares encefálicos hemorrágicos, mas também para outras patologias. Achados tomográficos ${ }^{2}$ também apóiam esta idéia, visto que quanto maior o distúrbio da consciência, maiores as chances de serem encontradas alteraçōes na TC, e pior prognóstico; distúrbio de consciência está associado a edema encefálico e quanto maior este for, maior o dano à barreira hematoencefálica e quanto maior o edema encefálico, maior o comprometimento da consciência.

\section{CONCLUSÓES}

A análise dos resultados deste estudo permitem concluir que: (1) A taxa de proteínas totais do LCR é um índice de prognóstico nos acidentes vasculares encefálicos hemorrágicos, em relação a sobrevida: quanto maior a taxa de proteínas totais, pior o prognóstico. (2) Todos os pacientes com taxa de proteínas totais no LCR acima de $3.000 \mathrm{mg} / 100 \mathrm{ml}$ faleceram. (3) Quando as taxas de proteínas são semelhantes, a idade e o sexo não influenciam o prognóstico em relação à sobrevida. (4) Homens acima de 50 anos de idade com acidente vascular encefálico hemorrágico têm taxas de proteínas totais mais elevadas que homens com menos de 50 anos.

\section{REFERENCIAS}

1. Adams RD, Victor MA. Principles of Neurology. Ed 3. New York: Intemational, 1986, p 569-640.

2. Benain J. Hipertensive intracerebral hemorrhage in south american; progress in surgical treatment. In Mizukami M, Kogure K, Kanaya H, Yamamori Y (eds). Hipertensive intracerebral hemorrhage. New York: Raven Press, 1983, p 133-145.

3. Brismar J, Sundbarg G. Subarachnoid hemonhage of unknown origin: prognosis and prognostic factors. J Neurosurg 1985, 63: 349-354.

4. Cahill DW, Ducker TB. Spontaneous intracerebral hemornhage. Clin Neurosurg 1982, 29: 722-729.

5. Cecotto C, De Nardi F, Schiavi F, Passoni B. Indicazioni e risultati del trattamento chirurgico degli ematomi intracerebrali spontanei. Minerva Med 1978, 69: 4393-4399.

6. Chehrazi BB, Giri S, Joy RM. Prostaglandins and vasoactive amines in cerebral vasospasm after aneurysmal subarachnoid hemorrhage. Stroke 1989, 20: 217-224.

7. Cochran WG. Some methods for strenghtering the common X2 test. Biometrics 1954, 10: 417-451.

8. Denis W, Ayer JB. Method for quantitative determination of protein in cerebrospinal fluid. Arch Intern Med 1920, 26: 436-442.

9. Dóczi T, Joó F, Adam G, Bojoky B, Szerdahelyi P. Blood-brain barrier damage during the acute stage of subarachnoid hemorrhage, as exemplified by a new animal model. Neurosurgery 1986, 18: 1163-1169.

10. Dóczi T, Jo6 F, Sonkodi S, Adam G. Increased vulnerability of the blood-brain barrier to experimental subarachnoid hemorrhage in spontaneously hipertensive rats. Stroke 1986, 17: 498-500.

11. Dózi T, Nemessanyi Z, Szegvary Z, Huszka E. Disturbances of cerebrospinal fluid circulation during the acute stage of subarachnoid hemorrhage. Neurosurgery 1983, 12: 435-438. 
12. Dughly M, Dropesh VP. Hipoglycorrhaquia does not necessarily indicate infection. BJCP 1989, 43: 182-183.

13. Fein JM. Hypertension and the central nervous system. Clin Neurosurg 1982, 29: 666-721.

14. Ferri-de-Barros JE, Bacheschi LA. Hemorragia intraparenquimatosa cspontânea: I. Conceito, incidência, etiologia e classificaçăo. Arq Bras Neurocir 1983, 2: 291-299.

15. Findlay $G$. Lumbar puncture in spontaneous subarachnoid haemorrhage. Br Med J 1982, 285: 1659.

16. Fischer $M$. Lumbar puncture and $C T$ in subarachonid hemorrhage. Neurology 1984, 34: 1400.

17. French KJ, Glasgow LG. Lumbar puncture in subarachonid haemorrhage: yes or no? N Z Med J 1985, 22: 383-384.

18. Hollander M, Wolf DA. Non parametric statistical methods. New York: Wiley, 1973. Computed tomography and the early diagnostic lumbar puncture. Can Med Assoc J 1979, 121: 150-151.

19. Humphreys RP. Computed tomography and the early diagnostic lumbar puncture. Can Med Assoc J 1979, 121: $150-151$.

20. Ivan LP. Computed tomography and early diagnostic lumbar puncture. Can Med Assoc J 1979, $121: 1442$.

21. McKissoc W, Richardson A, Walsh L. Primary intracerebral hemorrhage: results of surgical treatment in 244 consecutive cases. Lancet 1959, 2: 683-686.

22. Melaragno R Filho, Sanvito WL. Doenças vasculares do encefálo. São Paulo: Manole, 1975, p 236-245.

23. Nicholls ES, Jung J, Davies JW. Cardiovascular disease mortality in Canada. Can Med Assoc J 1981, 125: 981-992.

24. Olle H. Subarachnoid hemorrhage: prognosis when angiography revels no aneurysm: a report of 138 cases. Acta Med Scand 1958, 162: 493-503.

25. Poungvarin N, Viriyavejakul A. Spontaneous supratentorial intracerebral haemorrhage: a prognositc study. J Med Assoc Thai 1990, 73: 206-211.

26. Reis JB, Bei A, Reis-Filho JB. Líquido cefalorraquiano. São Paulo: Sarvier, 1980, pp 35-47, 49-61, 104-124.

27. Reis JB, Reis-Filho JB. O líquido cefalorraqueano no estudo dos pacientes com traumatismo craniencefálico. Rev Paul Med 1973, 81: 13-16.

28. Sasaki T, Kassel NF, Yamashita M, Fujiwara S, Zuccarello M. Barrier disruption in the major cerebral arteries following experimental subarachnoid hemorthage. J Neurosurg 1985, 63: 433-440.

29. Sasaki T, Kassel NF, Zuccarello M, Nakagomi T, Fujiwara S, Colohan ART, Lehman N. Barrier disruption in the major cerebral arteries during the acute stage after experimental subarachnoid hemorrhage. Neurosurgery 1986, 19: 177-184.

30. Shephard HR. Ruptured cerebral aneurysm: early and late prognosis with surgical treatment. A personal series, 1958-1980. J Neurosurg 1983, 59: 6-15.

31. Siegel S. Estadistica no paramétrica aplicada a las ciencias de la conducta. México: Trillas, 1975.

32. Tanaka H, Tanaka Y, Hayashi M. Secular trends in mortality for cerebrovascular disease in Japan, 1960 1979. Stroke 1982, 13: 574-581.

33. Teddy PJ. Lumbar puncture in spontaneous subarachnoid hemorrhage. Br Med J 1983, 286:143.

34. Tesementzis SA, Hitchock R, De Cothi A, Gill J. Comparative studies of the diagnostic value of cerebrospinal fluid spectrophotometry and computed tomographic scanning in subarachnoid hemorthage. Neurosurgery 1985, 17: 908-911.

35. Vermeulen M, van Gijn J, Blijenberg BG. Spectrophotometric analysis of CSF after subarachnoid hemorrhage: limitations in the diagnosis of rebleeding. Neurology 1983, 33: 112-114.

36. Volpato MM, Badia-Filho H, Vilanova LCP, Reis-Filho JB. Avaliação clínica e do LCR na meningencefalite tuberculosa da criança. In: Congresso Brasileiro de Neurologia 14, Rio de Janeiro, 1990. Temas Livres. Arq Neuropsiquiatr 1990, 48 (Suppl), resumo 225.

37. Weiss W, Figueroa W, Shapiro WH, Flippin HF. Prognostic fators in pneumococcal meningitis. Arch Intern Med 1967, 120: 517-524.

38. Wood JH. Neurobiology of cerebrospinal fluid. New York: Plenum, 1980, p185-190. 